\title{
Electromagnetic Position Sensing and Force Feedback for a Magnetic Stylus with an Interactive Display
}

\author{
Peter Berkelman, Member, IEEE, Bernadette Tix, and Hamza Abdul-Ghani
}

\begin{abstract}
This paper describes the design, implementation, validation, and demonstration of an electromagnetic system which can be incorporated into a graphical display to provide computer-controlled planar feedback forces on the tip of a stylus or fingertip-mounted magnet held near the display surface, according to the magnet position and virtual fixtures implemented in software. An array of magnetometer sensors is used to detect the position of the magnet, while a pair of box-shaped coils behind the display produces feedback forces on the stylus parallel to the plane of the display. Electromagnetic analysis for the system design is presented and system implementation is described. Validation results are given for force generation within a $100 \times 100 \mathrm{~mm}$ area and force interaction with a virtual obstacle is demonstrated.
\end{abstract}

\section{INTRODUCTION}

We have developed a system which can produce precisely controlled planar forces on a handheld stylus as it is manipulated near the surface of a display screen, using Lorentz forces produced by the interaction of currents in coils behind the display and a permanent magnet at the tip of the stylus. The position of the stylus tip is detected and calculated from a planar array of magnetometer sensors behind the display screen. With this system it is possible to implement haptic feedback forces corresponding to graphically displayed elements which can be directly physically felt by the user as the grasped stylus is manipulated to interact with virtual objects in the displayed environment.

Force feedback screens are an active area of development, as they can enhance interaction with a graphical environment in an immediate, intuitive manner through the haptic senses of the user [Banter, 2010]. Various technologies have been used to generate force feedback from a screen onto either the fingertip of the user or a grasped stylus, including ultrasound [Long et al., 2014] and electrostatic [Meyer et al., 2013] vibrations, actuated screens moving in lateral [Saga and Deguchi, 2012] or orthogonal [Sinclair et al., 2013] directions to the display plane, and styluses with internal actuation to generate forces against the screen [Kyung and Lee, 2008, Lee et al., 2004].

Electromagnetic forces are highly suitable to force feedback for interactive displays, because coils within the display can directly generate forces on a magnet in the stylus or at the fingertip, with no additional moving parts needed. A system

Peter Berkelman is with the Department of Mechanical Engineering at the University of Hawaii-Manoa, Honolulu, HI, 96822 USA e-mail: peterb@hawaii.edu.

Bernadette Tix is with the Information and Computer Sciences Department at the University of Hawaii-Manoa.

Hamza Abdul-Ghani is with the California Institute of Technology.

Manuscript received October, 2018.

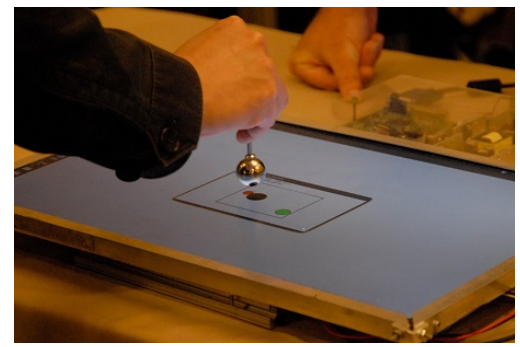

Fig. 1. Force feedback stylus in use

described in [Weiss et al., 2011] uses a planar array of electromagnets to generate attraction and repulsion on a fingertip magnet from a tabletop display. Design and performance issues involved with this and similar electromagnetic force feedback interactive surfaces are described in [Brink et al., 2014].

It is also possible, through detailed electromagnetic modeling of multiple coils acting together, to use an array of cylindrical coils to generate fully three-dimensional force and torque vectors in any direction on a rigid body containing two or more magnets. If a motion tracking system is used to sense position and orientation of the magnets in real time with a sufficient accuracy and update rate, then feedback control may be implemented to achieve stable magnetic levitation [Berkelman and Dzadovsky, 2010]. A magnetic levitation system using an array of cylindrical coils has been used to provide three-dimensional haptic feedback of force and torque on a grasped pen handle, co-located with a graphical display placed above the coils [Berkelman et al., 2012]. This system was further developed with a stereovision display with shutter glasses and head tracking for haptic interaction with dynamic simulated environments [Berkelman et al., 2013].

Other levitation devices have been developed which use flat coils embedded in a thin shell for actuation using Lorentz forces [Hollis and Salcudean, 1993]. Lorentz force levitation systems have been developed for force-reflecting teleoperation [S. Salcudean et al., 1995] and for haptic interaction [Berkelman and Hollis, 2000], commercialized as the Maglev 200 from Butterfly Haptics Inc.

The system described in the following sections has the novel features that both the position sensing and force generating elements are electromagnetic and contained within the display behind the screen, and that two coils alone are sufficient to generate forces in two dimensions across the area of the screen. Design analysis, validation of position sensing and force generation, and interactive demonstration results are presented. A preliminary demonstration of our system is shown in use with a display screen in Fig. 1. 


\section{LoRentz CoIL Design AND Modeling}

The Lorentz forces $F$ produced from current-bearing wires passing through magnetic fields are proportional to the flux density $B$, the wire length $L$, and the current $i$ :

$$
F=i \int d L \times B
$$

Therefore the force produced between a large flat current sheet and a cylindrical magnet above it with its axis of magnetization perpendicular to the sheet is in the plane of the sheet, perpendicular to the wire orientation, and depends only on the current and the magnetic field intersecting it. Furthermore, if two current sheets are layered together at right angles to one another as in [Berkelman, 2007], $x$ and $y$ forces in the plane of the fixed coils can be generated on the magnet, proportional to the current in each sheet.

Practical coil designs with finite dimensions cannot match the characteristics of the ideal case with current sheets of infinite planar dimensions however, due to effects at the edges of the current sheets, forces produced by the return paths of the currents, and forces produced by the return paths of magnetic flux from the stylus magnet. A practical design can minimize these effects through the use of box-shaped coils, with current return paths underneath the active areas of the coils at a distance sufficient to minimize Lorentz forces produced, while not resulting in excessive size or power consumption.

Numerical modeling using Radia [Chubar et al., 1998] software for a $9.5 \mathrm{~mm}$ thick, $20 \mathrm{~mm}$ diameter magnet with magnetization of 52.0 MGOe above a $180 \times 200 \times 40 \mathrm{~mm}$ box coil with $2.5 \mathrm{~A}$ of current, as pictured in Fig. 2, was used to find the $x, y$, and $z$ components of forces produced on the magnet depending on its position above the top current sheet. The forces produced in each direction for a magnet with a separation distance of $25 \mathrm{~mm}$ above the coil surface are shown in Figs. 3-5. These show that the $x$ forces produced parallel to the wire directions and the vertical $z$ forces are both within approximately $\pm 0.01 \mathrm{~N}$ in the $100 \times 100 \mathrm{~mm}$ center area of the coil, increasing towards the corners and edges of the coil, and the desired $y$ forces perpendicular to the wire directions vary between approximately $0.03 \mathrm{~N}$ and $0.06 \mathrm{~N}$ in a saddle shape. Therefore within a 100x100 mm effective area, the Lorentz forces from each coil are mostly in a single principal direction and $x y$ planar feedback forces can be generated by the two coils, disregarding the small forces produced by each coil in the vertical and wire directions within the effective area.

It is notable that the forces generated in Fig. 4 are less at the center and greater near the coil edges as the magnet is moved in the $y$ direction. The explanation for this is that the vertical components of the magnetic flux surrounding the magnet change direction at greater distances from the magnet axis, and where these areas of the magnetic field intersect the box coil Lorentz forces in the opposite direction are produced, thereby reducing the total force generated in the desired direction. When the magnet is near the edge of the coil, the area of the coil intersecting the magnetic flux lines in the opposite direction is reduced and larger forces are produced in the desired direction. Given the desired planar force vector to be generated and the magnet planar position, it is possible to calculate the needed current in each of the coil windings. In particular the force generated from one coil as shown in Fig. 4 can be approximated by a quadratic model in $x$ as

$$
f_{y}=0.4 I\left(0.037+0.000008537 x^{2}\right)
$$

with an $r^{2}$ value of 0.96 , where $I$ is the coil current in A.

The forces generated on the magnet also vary according to its distance from the coils and any tilt angle of the stylus axis relative to the surface normal, and these variations are not compensated by the force feedback system. In practice, the feedback forces on the stylus are easily perceptible by the fingertips when the magnet to screen distance is within approximately $15 \mathrm{~mm}$, although these decrease rapidly at larger distances. The variation of the feedback force direction as the stylus is tilted is not immediately perceptible to the user, as the force vector remains nearly perpendicular to the stylus and magnetization axis up to tilt angles of approximately 30 degrees.

\section{Stylus Location Sensing}

A 16x16 array of magnetometer Hall effect sensors, commercially available as the Basic GaussSense from GaussToys Inc., is used to sense and calculate the position and orientation of the stylus magnet [Liang et al., 2012]. Each sensor measures the vertical component of the flux density at its location, and the sensor voltages are digitized by a Teensy 2.0 microcontroller board and communicated to a host PC over a USB connection. Software is provided to calculate the position of the intersection of the central axis of magnetization of the stylus magnet with the sensing array. An approximate measurement of the magnet height above the array and its orientation is also provided. Various sizes, shapes, and magnetizations of cylindrical magnets may be used provided that their fields generated are within the sensing range of the sensor array.

This electromagnetic localization system works well as provided for many applications, however for our described system it was observed that the magnetic fields generated by currents in the coils interfere with the position sensing of the stylus magnet, causing errors in the sensed magnet position of several $\mathrm{mm}$ or more when force feedback is generated.

Fig. 6 shows uncalibrated sensor array data from the stylus magnet $30 \mathrm{~mm}$ above the top coil surface with a $2.5 \mathrm{~A}$ current in one coil, with the background sensor offset readings obtained with no coil current or magnet present subtracted from the actual sensor data measurements. Fig. 7 shows the result obtained from numerical modeling of the same conditions with Radia. These figures show that the magnetic fields produced by the box coil currents are much smaller that the fields from the magnet, and it is possible to accurately locate the magnet by disregarding sensor values below a given threshold. In practice, it was found that magnet position sensing errors can be practially eliminated by altering the localization software to disregard all sensor readings less than \pm 25 bits.

\section{IMPLEMENTATION AND VALIDATION}

The electromagnetic modeling results of the previous sections were used to implement a force-feedback system for 


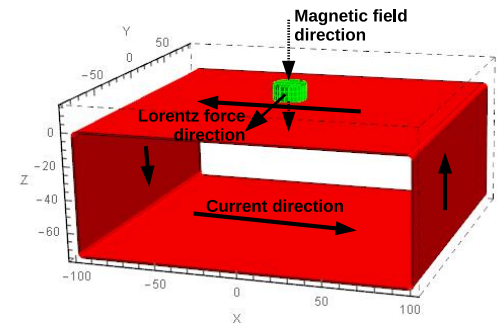

Fig. 2. Single box coil and magnet model

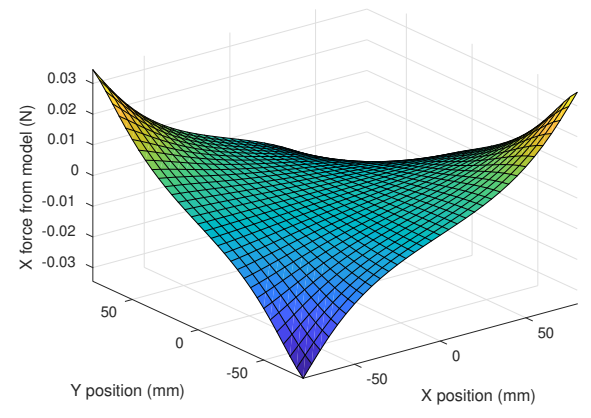

Fig. 3. Lorentz forces produced on stylus magnet in $\mathrm{X}$ direction

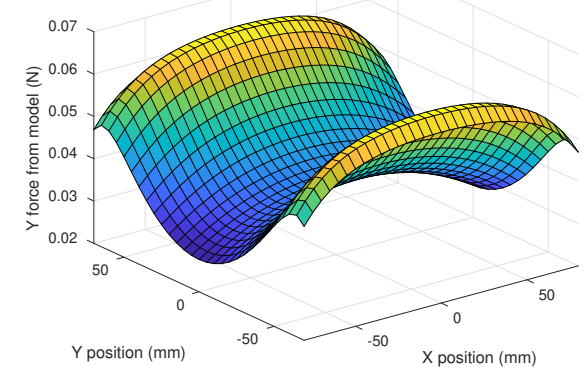

Fig. 4. Lorentz forces produced on stylus magnet in Y direction

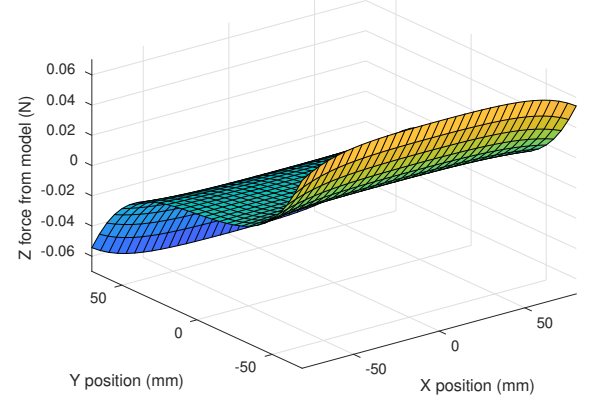

Fig. 5. Lorentz forces produced on stylus magnet in $\mathrm{Z}$ direction

a stylus magnet as described. Flat, box-shaped coils were wound on an aluminum frame in two layers in perpendicular directions, so that planar forces can be generated on a stylus magnet held near the top surface. The forces produced on the magnet in $X$ and $Y$ directions from a 2.5 A current in one of the coils were measured by strain gage load cells (Phidgets 100g Micro Load Cell) and are shown in Figs. 8 and 9, which correspond reasonably closely to the modeled results of Figs. 3 and 4, taking into consideration the limited accuracy of the strain gage force sensing.

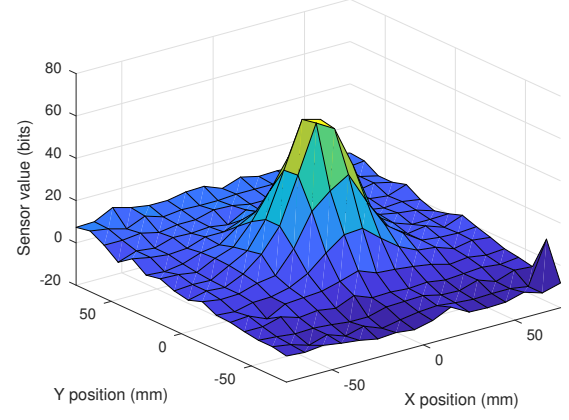

Fig. 6. Sensor values with $2.5 \mathrm{~A}$ in one coil and the magnet $25 \mathrm{~mm}$ above

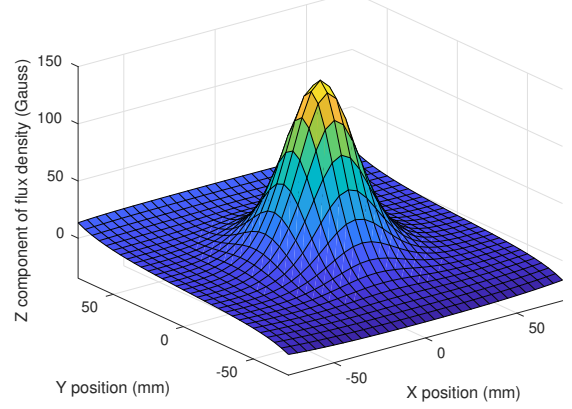

Fig. 7. Vertical component of magnetic field in the plane of the sensor array from Radia model, with $2.5 \mathrm{~A}$ in one coil and the magnet $25 \mathrm{~mm}$ above

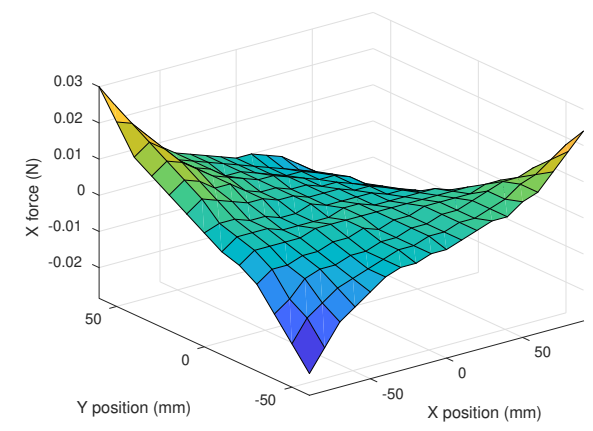

Fig. 8. Forces measured on stylus magnet in $\mathrm{X}$ direction

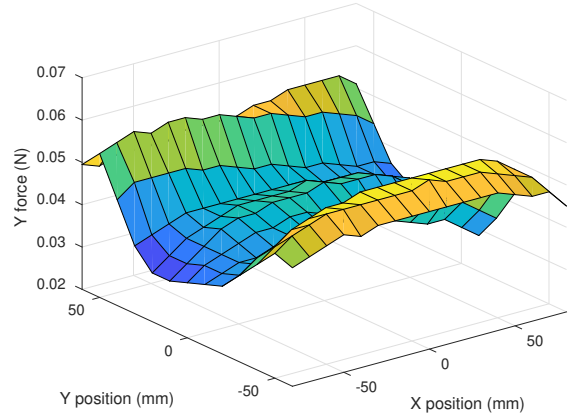

Fig. 9. Forces measured on stylus magnet in $\mathrm{Y}$ direction

The Gausssense magnetometer array is placed above the top of the coil box, as shown in Fig. 10. A thin video screen, with any iron-containing brackets and connectors removed, may be placed on top of the coils and magnetometers to form an interactive force-feedback display when used with a magnetic stylus. Two current amplifiers (Copley Controls 4212Z) are connected to a PC controller by USB through an analog output board (PhidgetAnalog 4-Output) to produce coil currents. The sensing, actuation, and interaction code is 


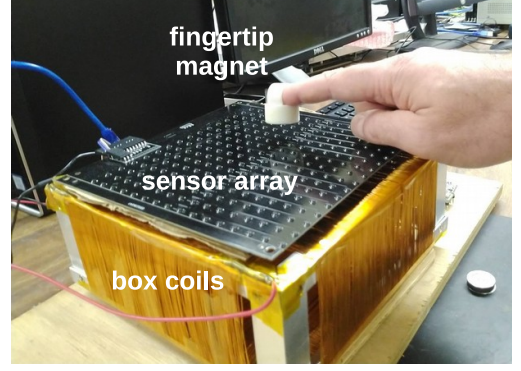

Fig. 10. System components
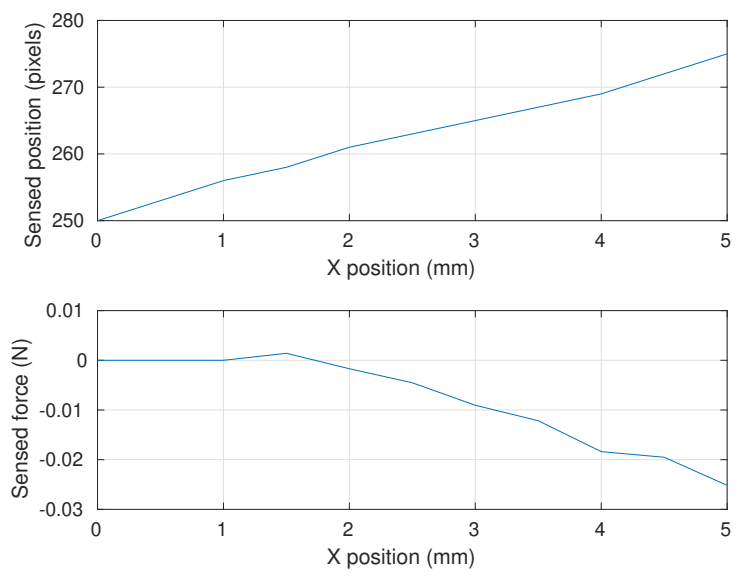

Fig. 11. Position and force results from haptic interaction

implemented in Processing 3.0 on a Linux PC. Equation (2) is used to calculate each coil current to be generated to produce the desired force components in $x$ and $y$ directions.

\section{SAMPLE INTERACTION Results}

Fig. 11 shows sensed position and force as the magnet is moved into a virtual obstacle by a motion stage. Position as measured in screen pixels was sensed and calculated from the GaussSense magnetometer sensor board and the feedback force was measured using a Phidgets strain gage load cell. The result shows increasing reaction forces generated on the magnet as it penetrates into the virtual object surface located at $1.5 \mathrm{~mm}$.

\section{CONCLUSION}

We have realized a system which delivers planar force feedback to a magnet fixed to a user stylus or fingertip in order to enable haptic force interaction with virtual objects and fixtures modeled in software. The system can be completely contained within a graphical display screen, uses both electromagnetic position sensing and force generation, and can be used with the display to constitute a combined, co-located graphic and haptic planar interface. Current and future development plans include further design refinement, user trials to evaluate the effectiveness of the haptic feedback, and including additional coils to increase the range of generated forces and so that vertical feedback forces may be generated in addition to planar forces.

\section{REFERENCES}

B. Banter. Touch screens and touch surfaces are enriched by haptic force-feedback. Information Display, 26(3):26-30, 2010.

P. Berkelman. A novel coil configuration to extend the motion range of lorentz force magnetic levitation devices for haptic interaction. In IEEE/RSJ International Conference on Intelligent Robots and Systems, San Diego, October 2007.

P. Berkelman and M. Dzadovsky. Novel design, characterization, and control method for large motion range magnetic levitation. IEEE Magnetics Letters, 1, January 2010.

P. Berkelman, M. Miyasaka, and J. Anderson. Co-located 3d graphic and haptic display using electromagnetic levitation. In IEEE Haptics Symposium, pages 77-81, Vancouver, March 2012.

P. Berkelman, S. Bozlee, and M. Miyasaka. Interactive rigid-body dynamics and deformable surface simulations with co-located maglev haptic and 3D graphic display. International Journal on Advances in Intelligent Systems, 6:289-299, 2013.

P. J. Berkelman and R. L. Hollis. Lorentz magnetic levitation for haptic interaction: Device design, function, and integration with simulated environments. International Journal of Robotics Research, 9(7):644-667, 2000.

J. B. Brink, A. J. Petruska, D. E. Johnson, and J. J. Abbott. Factors affecting the design of untethered magnetic haptic interfaces. In 2014 IEEE Haptics Symposium (HAPTICS), pages 107-114, Feb 2014.

O. Chubar, P. Elleaume, and J. Chavanne. A three-dimensional magnetostatics computer code for insertion devices. Journal of Synchrotron Radiation, 5:481-484, 1998.

R. L. Hollis and S. E. Salcudean. Lorentz levitation technology: a new approach to fine motion robotics, teleoperation, haptic interfaces, and vibration isolation. In International Symposium on Robotics Research, Hidden Valley, PA, October 1993.

K.-U. Kyung and J.-Y. Lee. wUbi-Pen: Windows graphical user interface interacting with haptic feedback stylus. In ACM SIGGRAPH 2008 New Tech Demos, SIGGRAPH '08, pages 42:1-42:4, New York, NY, USA, 2008.

J. C. Lee, P. H. Dietz, D. Leigh, W. S. Yerazunis, and S. E. Hudson. Haptic pen: A tactile feedback stylus for touch screens. In Proceedings of the 17th Annual ACM Symposium on User Interface Software and Technology, UIST '04, pages 291-294, New York, NY, USA, 2004. ACM.

R.-H. Liang, K.-Y. Cheng, C.-H. Su, C.-T. Weng, B.-Y. Chen, and D.-N. Yang. Gausssense: Attachable stylus sensing using magnetic sensor grid. In ACM Symposium on User Interface Software and Technology, UIST '12, pages 319-326, New York, NY, USA, 2012.

B. Long, S. A. Seah, T. Carter, and S. Subramanian. Rendering volumetric haptic shapes in mid-air using ultrasound. $A C M$ Transactions on Graphics, 33(6):181, November 2014.

D. J. Meyer, M. A. Peshkin, and J. E. Colgate. Fingertip friction modulation due to electrostatic attraction. In World Haptics Conference, pages 43-48, April 2013.

S. Salcudean, N.M. Wong, and R.L. Hollis. Design and control of a force-reflecting teleoperation system with magnetically levitated master and wrist. IEEE Transactions on Robotics and Automation, 11(2):844-858, December 1995.

S. Saga and K. Deguchi. Lateral-force-based 2.5-dimensional tactile display for touch screen. In 2012 IEEE Haptics Symposium (HAPTICS), pages 15-22. IEEE, 2012.

M. Sinclair, M. Pahud, and H. Benko. Touchmover: Actuated $3 \mathrm{~d}$ touchscreen with haptic feedback. In ACM International Conference on Interactive Tabletops and Surfaces, ITS '13, pages 287-296, New York, NY, USA, 2013.

M. Weiss, C. Wacharamanotham, S. Voelker, and J. Borchers. Fingerflux: Near-surface haptic feedback on tabletops. In $A C M$ Symposium on User Interface Software and Technology, UIST '11, pages 615-620, New York, 2011. ACM. 